\title{
Long-term effects on bone mineral density after four years of treatment with two intensive combination strategies, including initially high-dose prednisolone, in early rheumatoid arthritis patients: the COBRA-light trial
}

\author{
M. J. J. Lucassen ${ }^{1,2}$ - M. M. ter Wee ${ }^{1,2}$ (D) D. den Uyl ${ }^{2}$ - N. P. C. Konijn ${ }^{2}$ - M. T. Nurmohamed ${ }^{2,3}$ (D) A. E. Voskuyl ${ }^{2}$ (D) \\ D. van Schaardenburg ${ }^{3,4}$ (D) P. J. S. M. Kerstens ${ }^{5}$ - I. E. M. Bultink ${ }^{2}$ (D) M. Boers ${ }^{1}$ (D) W. W. Lems ${ }^{2,3}$ (D)
}

Received: 8 May 2020 / Accepted: 4 December 2020 / Published online: 19 January 2021

(C) The Author(s) 2021

\begin{abstract}
Summary In this study, no difference in bone loss was observed between patients with early RA initially treated with COmbinatietherapie Bij Reumatoide Artritis (COBRA) (including initially $60 \mathrm{mg}$ /day prednisolone) and patients treated with COBRA-light (including initially $30 \mathrm{mg}$ /day prednisolone) during 4-year observation.

Purpose To assess changes in bone mineral density (BMD) after 4 years in early rheumatoid arthritis (RA) patients initially treated with COBRA-light or COBRA therapy.

Methods In a 1 year, open-label, randomised, non-inferiority trial, patients were assigned to COBRA-light (methotrexate 25 mg/ week plus initially prednisolone $30 \mathrm{mg}$ /day) or COBRA (methotrexate $7.5 \mathrm{mg} /$ week, sulfasalazine $2 \mathrm{~g} /$ day plus initially prednisolone $60 \mathrm{mg} /$ day) therapy. After 1 year, antirheumatic treatment was at the discretion of treating rheumatologists. BMD was measured at baseline and after 1,2 and 4 years at hips and lumbar spine with dual-energy X-ray absorptiometry. BMD changes between treatment strategies on average over time were compared with GEE analysis.

Results Data from 155 out of 162 patients could be analysed: 68\% were female with a mean age of 52 (SD 13) years. Both COBRA-light and COBRA therapy showed declines in BMD at the total hip of $-3.3 \%$ and $-1.7 \%$, respectively $(p=0.12)$, and the femoral neck, $-3.7 \%$ and $-3.0 \%$, respectively $(p=0.95)$. At the lumbar spine, both treatment groups showed minor decline in BMD over 4 years: $-0.5 \%$ and $-1.0 \%$, respectively $(p=0.10)$.

Conclusion In a treat-to-target design in early RA, over 4 years, no differences between groups were found in change in BMD at total hip, femoral neck and the lumbar spine. At the hip, bone loss was around $3 \%$ in both groups, while mild bone loss was observed at lumbar spine, both in patients starting prednisolone 60 and $30 \mathrm{mg}$ /day. These data suggest that the well-known negative effects of prednisolone can be modulated by modern treatment of RA.
\end{abstract}

Keywords Rheumatoid arthritis · Early RA $\cdot$ Bone mineral density $\cdot$ COBRA-light $\cdot$ Combination therapy

M.J.J. Lucassen and M.M. ter Wee contributed equally to this work.

M. M. ter Wee

m.terwee@amsterdamumc.nl

1 Department of Epidemiology and Data Science, Amsterdam UMC, VU University Amsterdam, De Boelelaan 1089a, 1081 HV Amsterdam, The Netherlands

2 Department of Rheumatology and Clinical Immunology, Amsterdam Rheumatology and immunology Center, Amsterdam UMC, VU University Amsterdam, De Boelelaan, 1117 Amsterdam, Netherlands
3 Amsterdam Rheumatology and immunology Center, location Reade Rheumatology and Rehabilitation Center, Amsterdam, Netherlands

4 Department of Rheumatology and Clinical Immunology, Amsterdam Rheumatology and immunology Center, Amsterdam UMC, University of Amsterdam, Meibergdreef 9, Amsterdam, Netherlands

5 Department of Rheumatology, Westfriesgasthuis, Hoorn, Netherlands 


\section{Introduction}

Rheumatoid arthritis (RA) is a chronic, potentially disabling, autoimmune disorder, which not only affects the synovial tissue but also the bone structure itself. The inflammatory cascade, starting in the synovial tissue, results in a shift in osteoclast and osteoblast balance in favour of the osteoclasts. As a result, patients may develop both local bone loss, the so-called juxta-articular osteoporosis, erosions and generalised bone loss, which is associated with an increased fracture risk [1-5]. Osteoporosis is a well-known side effect of glucocorticoid (GC) treatment, especially at high doses, caused by a direct inhibition of bone formation and an early increase in bone resorption [6-10].

In the COmbinatietherapie Bij Reumatoide Artritis (COBRA)light trial, patients were treated with either COBRA-light (initially prednisolone $30 \mathrm{mg}$ /day and methotrexate (MTX) up to $25 \mathrm{mg}$ / week) or COBRA therapy (initially prednisolone $60 \mathrm{mg} /$ day, MTX $7.5 \mathrm{mg} /$ week and sulfasalazine $2 \mathrm{~g} /$ day (SSZ)). In this trial, COBRA-light proved to be as effective as COBRA in decreasing disease activity, after 1 and 4 years $[5,11]$. Since the negative effects of GCs on bone and fractures are dose related, some worries remained about the possible negative effects of the daily use of initially 30- or 60-mg prednisolone on bone [8, 12].

The aim of this sub-study was to investigate the difference in BMD changes over 4 years in early RA patients treated, with initially high-dose prednisolone during the first year, as part of either COBRA-light or COBRA therapy.

\section{Materials and methods}

\section{Study population}

All patients participated in the multicentre, open-label, randomised, non-inferiority COBRA-light trial. In- and exclusion criteria, randomisation process and study design of the COBRA-light trial (ISRCTN Clinical Trial Registration Number: 55552928) have been described earlier [7]. In short, patients with recent-onset RA, who were disease modifying antirheumatic drugs (DMARD) and prednisolone naive, were included from March 2008 to April 2011, in Amsterdam UMC, location VU University Medical Center, Reade Rheumatology and Rehabilitation Center, and Westfriesgasthuis Alkmaar, the Netherlands. Last patient visit was in April 2015. In total, 164 patients were randomised into two treatment strategies: COBRA-light or COBRA. Treatment goal was reaching minimal disease activity, defined as a disease activity score in 44 joints (DAS44) below 1.6 at every three-monthly visit. If the threshold of DAS $44<1.6$ was not met, therapy was intensified, either by increasing MTX in the COBRA group or starting MTX subcutaneous. If that was not sufficient to reach a DAS44 $<1.6$, etanercept was added at week 26 or 39 . Treatment steps in the first year were according to protocol. After 1 year, the treatment was at the discretion of the treating rheumatologist, usually aimed at clinical remission or minimal disease activity. Throughout the entire study, use of calcium/vitamin D supplements was at the discretion of the treating rheumatologist, whereas bisphosphonates were prescribed according to the guidelines for glucocorticoid-induced osteoporosis [7]. COBRA-light therapy proved to be non-inferior to COBRA therapy with respect to disease activity, functional outcome and radiographic progression in early RA patients, after 6 and 12 months of treatment $[5,7]$. The clinical records were examined from baseline until the 4-year visit to register duration, mean and cumulative dosage of prednisolone use.

The primary outcome of this sub-study was the difference in mean change in BMD at the lumbar spine between COBRA-light and COBRA therapy groups after 4 years. The secondary outcome measures were the mean differences in BMD change at the femoral neck and at the total hip between both treatment groups.

The medical ethics committee of the VU University Medical Center approved this study. All participating patients gave written informed consent, and the study was conducted in agreement with the Declaration of Helsinki/Good Clinical Practice.

\section{Bone mineral density}

BMD was measured by dual-energy X-ray absorptiometry (DXA) at the lumbar spine (L1-L4), total hip and femoral neck, according to protocol of the manufacturer. In total, 64 patients received a DXA scan on a Hologic Delphi (Hologic, Inc.: Bedford, MA, USA) equipment, used at location VU University Medical Center [13]. One hundred patients were scanned on a GE Lunar iDXA (GE Corporate, Madison, WI, USA) in Reade [13]. All patients had their baseline and subsequent scans on the same device, and each device was calibrated on a daily basis using a phantom. Results were expressed as absolute BMD in $\mathrm{g} / \mathrm{cm}^{2}$ and T-scores. A Tscore below $-2.5 \mathrm{SD}$, based on the lowest $\mathrm{T}$-score at lumbar spine or total hip, was defined as having osteoporosis [14]. BMD was measured at baseline, and after 1-year (T1), 2-year (T2) and 4-year follow-up (T4), the latter having a range of 34 to 74 months, and was not blinded to patients and imaging personnel, and collected as secondary outcome.

\section{Fractures}

Data on incident clinical fractures were prospectively collected during each visit, as secondary outcome. At the 4-year visit, $\mathrm{X}$-rays of the thoracic and lumbar spine were analysed according to Genant's method to identify morphometric vertebral fractures [15]. A reduction of at least $20 \%$ in vertebral height 
was considered a morphometric vertebral fracture. A vertebral fracture at baseline with a decrease of more than $10 \%$ in vertebral height after 4 years was considered a clinically relevant 'new morphometric vertebral fracture' during follow-up [11, 15]. Two assessors (IB, WFL) scored the X-rays, unaware of the treatment group. When no spine X-ray or an incomplete $\mathrm{X}$-ray was available for assessment, chest X-rays were used $[11,15]$.

\section{Statistical analysis}

Data are presented as mean (SD), percentage or median (interquartile range (IQR)) if the variable had a skewed distribution. To calculate the mean BMD values as well as the Tscores at each yearly visit, missing data was imputed according to the linear regression technique: the BMD measurement before and after the missing data was used to calculate the mean change over time between those two time periods. In case of a missing BMD value at baseline or week 208, the mean of the two BMD measurements at week 52 and 104 was used to calculate the missing value of week 208 by extracting the mean change from the week 104 BMD value and for week 0 by adding the mean change to the week 52 value. Patients were excluded from the analyses if more than two DXA scans were missing.

Generalised estimating equation (GEE) analysis with exchangeable correlation matrix was used to compare the differences in BMD changes between treatment strategies on average over time, allowing analyses of repeated measurements (three time moments). These analyses did not include the imputed data, as GEE analyses are developed to take into account missing data and adjust for these missing data accordingly. Analyses were corrected for bisphosphonate use over 4 years (yes versus no), cumulative GC use, age, gender and minimal disease activity defined as DAS44 < 1.6.

All statistical analyses were performed with IBM SPSS statistics V24.0 (IBM Corp., Armonk, NY, USA). A twosided $p<0.05$ was considered statistically significant.

\section{Results}

From the initial 164 patients in the COBRA-light trial, two patients did not initiate treatment because of withdrawal of informed consent directly after randomisation. These patients were not included in the intention-to-treat analyses. In total, $155(95 \%)$ patients had a 4-year follow-up with at least 2 DEXA scans, of which 76 received COBRA-light therapy and 79 COBRA therapy (Figs. 1). In the COBRA group, there were 62 patients with 4 scans, 13 patients with 3 scans, 4 patients with 2 scans, 2 patients with only 1 scan and 0 patients without any scan. In the COBRA-light group, these numbers were $64,12,1$ and 4, respectively. In total, 7 patients had none or only one BMD measurement and were therefore excluded from the analyses of this sub-study, and data of the patients with 1 or 2 missing DEXA scans were imputed. Of 13 patients (equally distributed between both strategies), some scans were technically inadequate, and data of these scans were also imputed. The mean (SD) age was 52 (13) years; 68\% were female. Baseline characteristics and demographic variables of both groups were similar, as shown in Table 1. After 4 years, the percentage of patients reaching minimal disease activity (DAS44<1.6) was $49 \%(n=34)$ in the COBRA-light and $50 \%(n=38)$ in the COBRA group.

\section{Change in BMD over 4 years}

The BMD at the total hip declined from 0.96 at baseline to 0.92 in COBRA-light after 4 years (see Fig. 2 and Supplementary file 1), which is equivalent to a $3.3 \%$ decrease in 4 years. Within the COBRA group, the decline was less: from 0.95 to 0.94 after 4 years, equivalent to a $1.7 \%$ decrease. The mean difference on BMD change over 4 years between the two groups at the total hip was -0.02 (95\% CI $[-0.04$; 0.00 ], $p=0.12$; see Table 2), corrected for bisphosphonate usage (yes versus no), cumulative prednisolone dosage, age, gender and disease activity based on DAS44 (DAS44 < 1.6 versus DAS44 $\geq 1.6$ ). At the lumber spine, the mean difference between the two groups was similar to that of the total hip, but with a proportion bone loss of $0.5 \%$ in the COBRAlight group and $1.0 \%$ in the COBRA group (Fig. 2). At the femoral neck, the amount of bone loss was larger: $-3.7 \%$ and $-3.0 \%$ for the COBRA-light and COBRA group, respectively, with an adjusted mean difference of -0.00 (95\% CI $[-0.02$; 0.02 ], $p=0.95$ ).

The results of the crude analyses can be found in Supplementary file 2 .

\section{Bisphosphonate and prednisolone use over 4 years}

The percentage of bisphosphonate use at any time during the trial period was $29 \%(n=21)$ for the COBRA-light group versus $23 \%(n=17)$ in the COBRA group $(p=0.45)$. At the 4-year visit, bisphosphonate use was only $11 \%(n=8)$ versus $4 \%(n=3)$ for COBRA-light and COBRA therapy, respectively $(p=0.11)$. Within the COBRA strategy, around $1 / 3$ of patients used calcium/vitamin D supplements compared to minimal use in the COBRA-light group in the first year of treatment. In years 2 to 4 , hardly any patients used calcium/ vitamin D supplements (data not shown).

The results of prednisolone use during the 4-year follow-up have been published before in Konijn et al. [11]. In short, after 1 year $41 \%(n=29)$ of the COBRA-light patients and $43 \%$ of the COBRA patients continued prednisolone $(p=0.83)$. After 4 years, these numbers were 19 and $22 \%$, respectively ( $p=$ 0.69). The cumulative median prednisolone dosage over the 


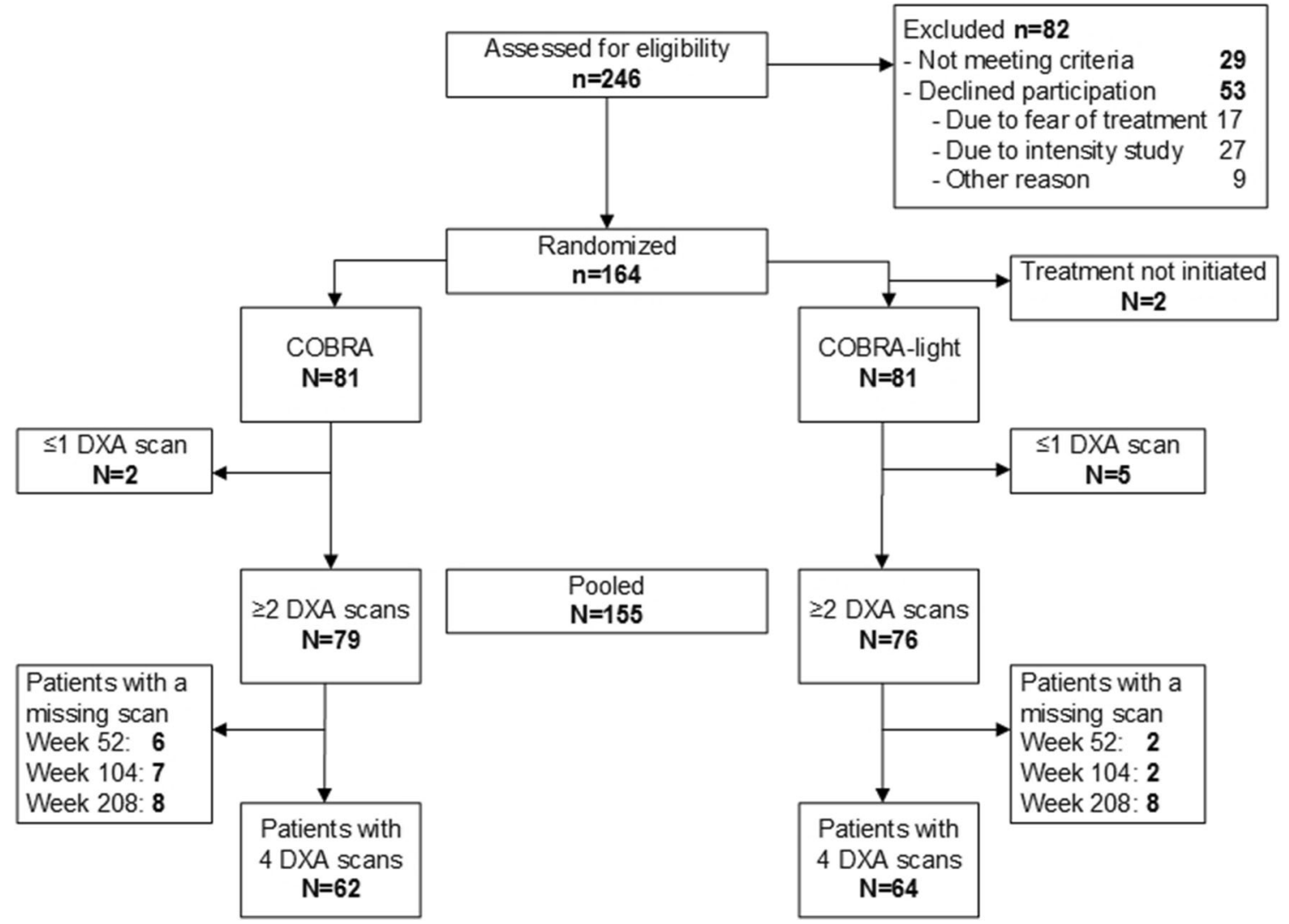

Fig. 1 Flow chart of Combinatietherapie Bij Reumatoïde Artritis (COBRA)-light trial. Adaption of Fig. 1 from ter Wee et al. (2014) (5) DXA, dualenergy X-ray absorptiometry; T4, measurement after 4 years

4-year follow-up period was $2.6 \mathrm{~g}$ (IQR 1.9-5.9) in the COBRA-light group and $3.2 \mathrm{~g}$ (IQR 2.5-6.2) in the COBRA group, with a median daily dose of $8.1 \mathrm{mg}$ (IQR 7.4-8.1) and $9.6 \mathrm{mg}$ (IQR 8.0-10.4), respectively (Table 3 ). The median duration of prednisolone use was 324 (IQR 239-899) and 348 (IQR 239-738) days, respectively [8]. These differences correspond to the difference in initial treatment strategy: after the first 9 weeks, both therapy groups had reduced the prednisolone dose according to protocol to $7.5 \mathrm{mg}$ per day. The median daily dose as of 10 weeks to 4 years was $6.2 \mathrm{mg}$ /day (IQR $6.2-$ 6.2) in COBRA-light therapy versus $6.4 \mathrm{mg}$ /day (IQR 6.17.0) in COBRA therapy, showing a nonsignificant minimal difference in prednisolone dosage during the follow-up period $(p=0.64)$.

There were no significant differences between both therapy groups in cumulative prednisolone dosage $(p=0.39)$ or the amount of days using prednisolone $(p=0.85)$, although the median daily prednisolone dose did significantly differ between both groups $(p<0.005)$, in favour of COBRA-light. Of the patients still on prednisolone at 4 years, the median daily dose after 4 years did not significantly differ: $7.6 \mathrm{Mg} /$ day (IQR 6.7-7.9) in the COBRA-light group and $7.5 \mathrm{mg} /$ day (IQR 6.9-8.4) in the COBRA group $(p=0.65)$. The median cumulative prednisolone dosage was $9.0 \mathrm{~g}$ (IQR 7.8-11.1) in

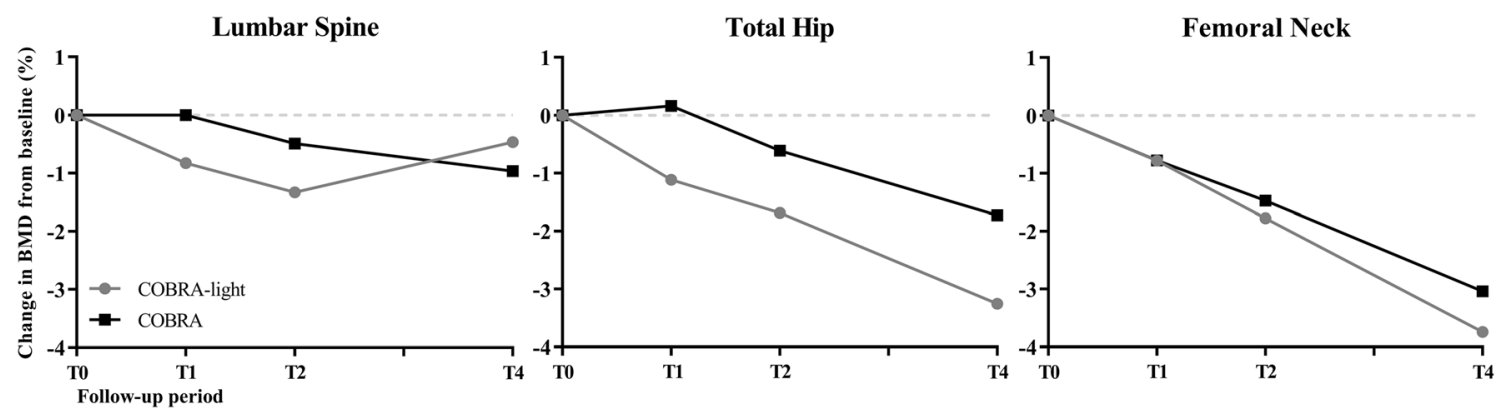

Fig. 2 Mean changes in BMD over 4 years at the total hip, femoral neck and lumbar spine. Data are expressed as mean percentage from baseline. Grey, COBRA-light therapy; black, COBRA therapy. $N=68$ or greater at all time points. COBRA, Combinatietherapie Bij Reumatoïde Artritis; $\mathrm{BMD}$, bone mineral density; $\mathrm{T} 1$, measurement after 1 year; $\mathrm{T} 2$, measurement after 2 years; T4, measurement after 4 years 
the COBRA-light group and $10.1 \mathrm{~g}$ (IQR 7.1-13.1) in the COBRA group $(p=0.83)$.

\section{Fractures and osteoporosis}

Of the 155 patients included in this sub-study, 21 patients had no recent X-ray available with a follow-up of at least 3 years after inclusion. Therefore, fracture data was only available in 134 patients (63 COBRA-light and 71 COBRA). In the COBRA-light therapy group, 11 patients $(17 \%)$ with a nonvertebral fracture were documented compared to seven $(11 \%)$ in the COBRA therapy group after 4 years $(p=0.21$; Table 3). In both groups, eight patients with one new morphometric vertebral fractures were found. In the COBRA-light therapy group, two (3\%) patients had a clinical vertebral fracture, and one patient in the COBRA-light therapy group had both a vertebral and a nonvertebral fracture. Of 5 patients (8\%) in the COBRA-light group, only lateral chest X-rays at baseline were present, compared to $10(14 \%)$ in the COBRA group. Of these patients, 3 in the COBRA-light group and 6 in the COBRA group had one or two newly diagnosed thoracic or lumbar fractures during the follow-up period. The total number of patients with a nonvertebral and vertebral fracture was 19 in the COBRA-light group versus 14 in the COBRA group $(p=0.17)$.
There was no significant difference in bone loss at the lumbar spine $(p=0.99)$, total hip $(p=0.77)$ or femoral neck $(p=0.81)$ between patients having a nonvertebral fracture during follow-up or not, irrespectively of treatment group. There were also no differences between therapy groups in possible known risk factors for fractures from the literature [16-18]: age $(p=0.43)$, BMI $(p=0.40)$, previous fractures $(p=0.17)$, menopausal state $(p=0.32)$, currently smoking $(p=0.49)$, a history of smoking $(p=0.84)$ or having at least one fall during follow-up $(p=0.36)$.

At all follow-up measurements, there were no significant differences in the prevalence of osteoporosis, defined as a $\mathrm{T}$-score below $-2.5 \mathrm{SD}$ at the spine and/or the hip, between both treatment groups (Table 3 ). Over 4 years, clinically relevant bone loss (defined as a predefined arbitrary difference as more than $5 \%$ bone loss, based on literature [6]) was found at the total hip in $28 \%(n=21)$ of the COBRA-light-treated patients versus $26 \%(n=20)$ of the COBRA-treated patient $(p=0.78)$. At the lumbar spine, more than $5 \%$ bone loss was found in $31 \%(n=$ $23)$ versus $27 \%(n=21)$, respectively $(p=0.58)$, and $41 \%$ $(n=31)$ versus $37 \%(n=29)$, respectively at the femoral neck $(p=0.60)$. When pooling all patients, clinically relevant bone loss after 1 year was found in $1.3 \%$ of the 155 patients at the total, $8.9 \%$ at the lumbar spine, and $7.7 \%$ at the femoral neck.
Table 1 Demographic variables at baseline

\begin{tabular}{lll}
\hline & COBRA-light $n=76$ & COBRA $n=79$ \\
\hline Female, $n(\%)$ & $52(68)$ & $53(67)$ \\
Age, years & $51(13)$ & $53(13)$ \\
Disease duration, weeks, median (IQR) & $16(8-35)$ & $16(9-28)$ \\
RF positive, $n(\%)$ & $43(57)$ & $45(57)$ \\
Anti-CCP positive, $n(\%)$ & $50(66)$ & $50(63)$ \\
Erosions, $n(\%)$ & $2(3)$ & $5(6)$ \\
DAS44-score & $4.0(0.93)$ & $4.1(0.75)$ \\
DAS28-score & $5.3(1.2)$ & $5.6(1.1)$ \\
HAQ-score & $1.3(0.73)$ & $1.4(0.64)$ \\
BMI, median (IQR) & $24.6(21.2-28.5)$ & $24.7(21.9-26.9)$ \\
Familiar history of osteoporosis, $n(\%)$ & $15(20)$ & $18(23)$ \\
Smoking, $n(\%)$ & $20(26)$ & $24(30)$ \\
Previous fractures, $n(\%)$ & $21(28)$ & $30(38)$ \\
Alcohol use, $n(\%)$ & $59(78)$ & $57(72)$ \\
Calcium intake, mg/day, median (IQR) & $765(585-1050)$ & $765(585-920)$ \\
Postmenopausal status, $n(\%)$ of women & $24(59)$ & $31(69)$ \\
T-score lumbar spine & $-0.61(1.2)$ & $-0.44(1.4)$ \\
T-score total hip & $-0.51(0.99)$ & $-0.53(1.1)$ \\
T-score femoral neck & $-0.81(0.95)$ & $-0.72(1.1)$ \\
\hline
\end{tabular}

Data are presented as mean (SD) unless otherwise stated

$\mathrm{RF}$, rheumatoid factor; anti-CCP, anti-cyclic citrullinated peptide; DAS44, 44-joint count disease activity score; DAS28, 28-joint count disease activity score; HAQ, Health Assessment Questionnaire; BMI, body mass index; $\mathrm{SD}$, standard deviation; IQR, interquartile range 


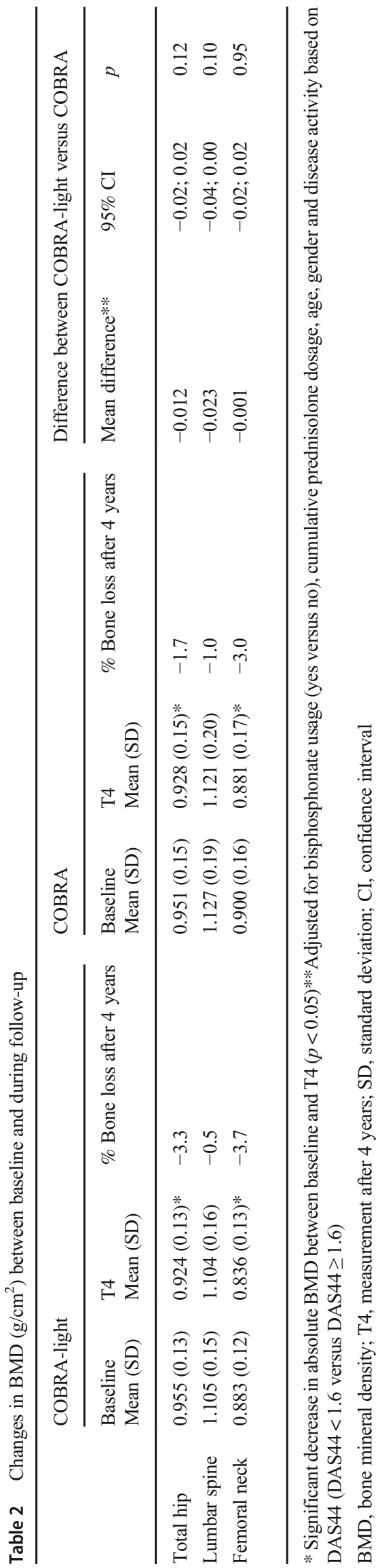

\section{Discussion}

This 4-year trial follow-up study showed that in early RA patients initially treated with intermediate $(30 \mathrm{mg} /$ day $)$ or high-dose $(60 \mathrm{mg} /$ day $)$ prednisolone, no significant difference was found in change in BMD at the hips and spine: $3 \%$ bone loss at the hips and mild bone loss $(0.5-1.0 \%)$ at the lumbar spine. This finding is consistent with results of other studies reporting more pronounced bone loss in the hip as compared to lumbar spine in RA patients treated with GC [6, 19-21].

The overall limited bone loss over 4 years in early RA patients treated with GCs, demonstrated in this study, is remarkable. It has been suggested that the negative effects of prednisolone on the bone mass in RA patients are counteracted by their favourable suppressive effects on the activity of the underlying disease, which might explain the limited bone loss occurring during both treat-to-target designs with initially high-dose prednisolone [21-24]. Another factor that also might have contributed to the limited bone loss is the on average short disease duration of only 16 weeks (IQR 9.030.0 ) and a relatively young patient population with a mean age of 52 (SD 13) years. The limited bone loss found in this study was similar with the average bone loss of postmenopausal women, around $1-2.5 \%$ per year at the femoral neck and $1-2 \%$ per year at the lumbar spine, as $64 \%$ of women in this patient population were postmenopausal at baseline $[25$, 26]. Before starting the study, bone loss of $5 \%$ or more was arbitrary defined as clinically relevant in this study. We observed more than $5 \%$ bone loss at the total hips in 28 and $26 \%$ of the patients and at the lumbar spine in 31 and $27 \%$ of the patients, in the COBRA-light and COBRA group, respectively. Although this seems to be substantial, it is important to realize that two-thirds of our patients were women, with a mean age of 52 years, thus early postmenopausal women with active RA at baseline, and that we observed these patients over 4 years.

Comparison with other studies concerning BMD changes during COBRA therapy is speculative because of differences in follow-up duration, study design and study population between studies. However, the results of the present COBRAlight study seem to be in line with the results of the original COBRA study $[6,27]$, reporting a slightly higher decline in BMD at the femoral neck $(-1.9 \%)$ after 56 weeks of COBRA therapy [6] and a mean reduction in BMD at the lumbar spine of $-1.3 \%$ (95\% CI [-2.3; 0.4]) [27]. In the present study, the mean decline in BMD over 4 years at the lumbar spine seemed to be smaller, probably due to the more aggressive treat-totarget strategy and attention to bone saving therapies such as bisphosphonates. Although the difference in the percentage of calcium/vitamin D users in the first year was substantial, we do not think that this difference had a large effect on the changes in BMD, as the effects of prednisolone, disease activity and bisphosphonates on BMD are much stronger. 
Table 3 Prevalence of osteoporosis, incidence of fractures over 4 years and prednisolone use

\begin{tabular}{llll}
\hline & COBRA-light $(n=76)$ & COBRA $(n=79)$ & $p$ \\
\hline Osteoporosis & & & \\
Baseline & $5(7)$ & $5(6)$ & 0.97 \\
After 1 year & $6(8)$ & $5(6)$ & 0.70 \\
After 4 years & $8(11)$ & $7(9)$ & 0.73 \\
Prednisolone use over 4 years & & & \\
Use of prednisolone & $14(20)$ & $17(22)$ & 0.76 \\
Cumulative dose, g & 2.6 & 3.2 & 0.39 \\
Median daily dose, mg/day & 8.1 & 9.6 & $<0.005^{+}$ \\
Median daily dose week 10 to 4 years, mg/day & 6.2 & 6.4 & 0.64 \\
Fractures & $\boldsymbol{n}=\mathbf{6 3}$ & $\boldsymbol{n}=\mathbf{7 1}$ & \\
Baseline, in history & $21(33)$ & $30(42)$ & 0.17 \\
Nonvertebral fractures, during first year & $3(4)$ & $2(3)$ & 0.64 \\
Nonvertebral fractures, during 4 years & $11(17)$ & $7(10)$ & 0.21 \\
Morphometric vertebral fractures, during 4 years & $8(13)$ & $8(11)$ & 0.80 \\
Clinical vertebral fractures, during 4 years & $2(3)$ & $0(0)$ & 0.14 \\
Number of patients with a fracture, during 4 years & $19(30)$ & $14(20)$ & 0.17 \\
\hline
\end{tabular}

Data are presented as number (percentage), unless stated otherwise

${ }^{+}$Significant difference between COBRA-light and COBRA therapy groups, Data are presented as $n(\%)$ unless otherwise stated. Data under Fractures are based on the $\mathrm{n}$ of patients who reported fractures

g, grams; mg, milligrammes
Compared to other studies on the influence of a treat-to-target design on bone mass in early RA patients, bone loss at the total hip and the lumbar spine found in this study were similar to the findings of the BeSt study [28]: an average bone loss of $-1.0 \%$ at the lumbar spine and $-0.8 \%$ at total hip after 1 year. Haugeberg et al. reported a bone loss of -2.9 to $-4.0 \%$ at the hip after 5 years and only $-0.5 \%$ at the lumbar spine [20], while the mean prednisolone dose used ( $5 \mathrm{mg}$ /day) was lower than in our study. The discrepancy between the relatively small changes in BMD of the spine and the larger changes in BMD at the hip might be explained by the coexistence of other factors which increase BMD at the lumbar spine: spondylosis, aortic atherosclerosis and, although less frequent, lumbar vertebral fractures. Furthermore, in the present study, comparable BMD change was demonstrated in patients receiving initially a higher dose of prednisolone compared to a lower dose prednisolone, which finding highlights a minimal increase of the adverse effect on bone of initial high-dose prednisolone therapy.

Of our patients, 6 to $7 \%$ had osteoporosis at baseline. This frequency is well below the average prevalence of osteoporosis demonstrated in the elderly general Western population: approximately $21 \%$ in women aged $50-84$ years [29, 30]. This might be due to the relatively young patient group with a short disease duration in this study, the initiation of treatment early in the disease course and use of combination drug therapy in a treat-to-target design. Bisphosphonate use at any moment over these 4 years was $29 \%$ of COBRA-light therapy patients and $23 \%$ of COBRA therapy patients, which was prescribed at the discretion of the treating rheumatologist.

In 4 years, no differences in fracture incidence were found between therapy groups, both in nonvertebral and vertebral fractures. In COBRA-light therapy group, 10 (13\%) patients had a nonvertebral fracture, compared to $6(8 \%)$ in the COBRA therapy group $(p=0.21)$. Since nonvertebral fractures are often related to trauma, this small difference between therapy groups might be due to chance.

The significant difference found between therapy groups in median daily prednisolone dose during 4 years of follow-up was most likely due to the difference in starting dose between COBRA and COBRA-light treatment schedules, as there was no difference between therapy groups in median daily prednisolone dose from 10 weeks to 4 years, which might explain the absence of differences in BMD changes between the two groups. Patients who still used prednisolone after 4 years generally used a lower daily dose of prednisolone than $7.5 \mathrm{mg} /$ day.

The strength of this manuscript includes the length of follow-up which is unique in RA patients treated with GC and the very high retention of patients (155 out of 162 patients, 95\%) in this long-term follow-up study. But some limitations need to be taken into consideration. First of all, two different DXA scan manufactures were used. It was shown that the data from these two machines were comparable at the hip level but significantly different at other places [13]. Patients in our trial were always measured on the same device, 
making follow-up data comparable, so our conclusions remain the same as differences at the hip were minimal between both treatment groups. Secondly, bisphosphonates were not prescribed to all patients by protocol but only at the discretion of the treating rheumatologist. As a consequence, in this group of relatively young patients having a short disease duration, bisphosphonates were prescribed to a minority of the patients (29\% in the COBRA-light group and 23\% in the COBRA group). That bisphosphonate use did not influence the results of the GEE analysis significantly might thus be a power issue. Thirdly, baseline assessment of vertebral fractures was performed using lateral chest X-rays instead of lateral thoracolumbar spine X-rays in a few patients due to the fact that that lateral spine $\mathrm{X}$-rays were missing or incomplete in these patients. It is possible that baseline fractures were missed, which did occur in the follow-up period.

\section{Conclusion}

In this study, no difference in bone loss was observed between patients with early RA initially treated with COBRA and patients treated with COBRA-light during 4-year observation. Bone loss was mild over 4 years, emphasising that modern treatment of RA in a treat-to-target design can limit the usually occurring elevated bone loss in early RA, even when patients are initially treated with prednisolone.

Supplementary Information The online version contains supplementary material available at https://doi.org/10.1007/s00198-020-05781-7.

Acknowledgements We would like to thank all patients for their participation in this study, all physicians who enrolled patients in this study and all research nurses who were involved in patient management. Also, we would like to thank Mrs. B.S. Blomjous and Mrs. E. Kooijmans for entering all data into the COBRA-light therapy database.

Funding This research was performed within the framework of project T1-106 of the Dutch Top Institute group, and additionally funded by an unrestricted grant from Pfizer [grant number: WS905749].

Data availability There is no additional unpublished data from this study available for others to use. Data was published by den Uyl et al. (2014), ter Wee et al. (2015) and Konijn et al. (2017). (5, 7, 11).

\section{Compliance with ethical standards}

Conflicts of interest Mike Nurmohamed has received speaker fees and advisory board fees from Janssen, Roche, Merck Sharp \& Dohme, Pfizer, Eli Lilly, Bristol-Myers Squibb and Abbvie. Irene Bultink has received speaker fees or advisory board fees from Lilly Netherlands, Merck Sharp \& Dohme, Amgen, UCB, Roche Netherlands, and Sanofi Genzyme. Maarten Boers has received consultancy fees from Pfizer, UCB and Teva. Willem Lems has received speaker fees and advisory boards from Eli Lilly, Amgen, Pfizer, UCB, Abbvie and Roche and an educational grant from Pfizer.

All other authors have declared no conflicts of interest.
Ethics approval Medical Ethics Committees at each participating centre approved the protocol; patients gave written informed consent before inclusion, and the study was conducted in accordance with the Declaration of Helsinki/Good Clinical Practice.

Open Access This article is licensed under a Creative Commons Attribution-NonCommercial 4.0 International License, which permits any non-commercial use, sharing, adaptation, distribution and reproduction in any medium or format, as long as you give appropriate credit to the original author(s) and the source, provide a link to the Creative Commons licence, and indicate if changes were made. The images or other third party material in this article are included in the article's Creative Commons licence, unless indicated otherwise in a credit line to the material. If material is not included in the article's Creative Commons licence and your intended use is not permitted by statutory regulation or exceeds the permitted use, you will need to obtain permission directly from the copyright holder. To view a copy of this licence, visit http://creativecommons.org/licenses/by-nc/4.0/.

\section{References}

1. Vosse D, de Vlam K (2009) Osteoporosis in rheumatoid arthritis and ankylosing spondylitis. Clin Exp Rheumatol 27:S62-S67

2. van Staa TP, Leufkens HG, Cooper C (2002) The epidemiology of corticosteroid-induced osteoporosis: a meta-analysis. Osteoporos Int 13:777-787

3. Geusens P, Lems WF (2011) Osteoimmunology and osteoporosis. Arthritis Res Ther 13:242

4. Clarke B (2008) Normal bone anatomy and physiology. Clin J Am Soc Nephrol 3(Suppl 3):S131-S139

5. ter Wee MM, den Uyl D, Boers M, Kerstens P, Nurmohamed M, van Schaardenburg D et al (2015) Intensive combination treatment regimens, including prednisolone, are effective in treating patients with early rheumatoid arthritis regardless of additional etanercept: 1-year results of the COBRA-light open-label, randomised, noninferiority trial. Ann Rheum Dis 74:1233-1240

6. Boers M, Verhoeven AC, Markusse HM, van de Laar MA, Westhovens R, van Denderen JC et al (1997) Randomised comparison of combined step-down prednisolone, methotrexate and sulphasalazine with sulphasalazine alone in early rheumatoid arthritis. Lancet. 350:309-318

7. den Uyl D, ter Wee M, Boers M, Kerstens P, Voskuyl A, Nurmohamed M et al (2014) A non-inferiority trial of an attenuated combination strategy ('COBRA-light') compared to the original COBRA strategy: clinical results after 26 weeks. Ann Rheum Dis 73:1071-1078

8. Homik J, Cranney A, Shea B, Tugwell P, Wells G, Adachi R et al (2000) Bisphosphonates for steroid induced osteoporosis. Cochrane Database Syst Rev (2):CD001347

9. van der Goes MC, Jacobs JW, Boers M, Andrews T, Blom-Bakkers MA, Buttgereit F et al (2010) Patient and rheumatologist perspectives on glucocorticoids: an exercise to improve the implementation of the European league against rheumatism (EULAR) recommendations on the management of systemic glucocorticoid therapy in rheumatic diseases. Ann Rheum Dis 69:1015-1021

10. van der Goes MC, Jacobs JW, Boers M, Andrews T, Blom-Bakkers MA, Buttgereit $\mathrm{F}$ et al (2010) Monitoring adverse events of lowdose glucocorticoid therapy: EULAR recommendations for clinical trials and daily practice. Ann Rheum Dis 69:1913-1919

11. Konijn NPC, van Tuyl LHD, Boers M, den Uyl D, Ter Wee MM, van der Wijden LKM et al (2017) Similar efficacy and safety of initial COBRA-light and COBRA therapy in rheumatoid arthritis: 
4-year results from the COBRA-light trial. Rheumatology. 56: $1586-1596$

12. Güler-Yuksel M, Hoes JN, Bultink IEM, Lems WF (2018) Glucocorticoids. Inflammation and Bone Calcif Tissue Int 102: 592-606

13. Wilson KE, Smith AP Monitoring BMD with DXA: Short- and Long-term Precision. Website Hologic Education. Assessed on 24 March 2020. https://hologiced.com/wp-content/uploads/2018/06/ Wilson-KE-and-Smith-AP.-Monitoring-BMD-with-DXA-W-157. pdf

14. Kanis JA (1994) Assessment of fracture risk and its application to screening for postmenopausal osteoporosis: synopsis of a WHO report. WHO Study Group. Osteoporos Int 4:368-381

15. Genant HK, Wu CY, van Kuijk C, Nevitt MC (1993) Vertebral fracture assessment using a semiquantitative technique. J Bone Miner Res 8:1137-1148

16. Cooper C, Coupland C, Mitchell M (1995) Rheumatoid arthritis, corticosteroid therapy and hip fracture. Ann Rheum Dis 54:49-52

17. Weinstein RS (2011) Clinical practice. Glucocorticoid-induced bone disease. N Engl J Med 365:62-70

18. Qaseem A, Forciea MA, McLean RM, Denberg TD (2017) Treatment of low bone density or osteoporosis to prevent fractures in men and women: a clinical practice guideline update from the American College of Physicians. Ann Intern Med 166:818-839

19. Sambrook PN, Eisman JA, Yeates MG, Pocock NA, Eberl S, Champion GD (1986) Osteoporosis in rheumatoid arthritis: safety of low dose corticosteroids. Ann Rheum Dis 45:950-953

20. Haugeberg G, Helgetveit KB, Forre O, Garen T, Sommerseth H, Proven A (2014) Generalized bone loss in early rheumatoid arthritis patients followed for ten years in the biologic treatment era. BMC Musculoskelet Disord 15:289

21. Lems WF, Baak MM, van Tuyl LH, Lodder MC, Dijkmans BA, Boers M (2016) One-year effects of glucocorticoids on bone density: a meta-analysis in cohorts on high and low-dose therapy. RMD Open 2:e000313

22. Vis M, Güler-Yüksel M, Lems W (2013) Can bone loss in rheumatoid arthritis be prevented? Osteoporos Int 24:2541-2553
23. Siu S, Haraoui B, Bissonnette R, Bessette L, Roubille C, Richer V, Starnino T, McCourt C, McFarlane A, Fleming P, Kraft J, Lynde C, Gulliver W, Keeling S, Dutz J, Pope JE (2015) Meta-analysis of tumor necrosis factor inhibitors and glucocorticoids on bone density in rheumatoid arthritis and ankylosing spondylitis trials. Arthritis Care Res 67:754-764

24. Santiago T, da Silva JA (2014) Safety of low- to medium-dose glucocorticoid treatment in rheumatoid arthritis: myths and reality over the years. Ann N Y Acad Sci 1318:41-49

25. Recker R, Lappe J, Davies K, Heaney R (2000) Characterization of Perimenopausal bone loss: a prospective study. J Bone Miner Res 15:1965-1973

26. Finkelstein JS, Brockwell SE, Mehta V, Greendale GA, Sowers MR, Ettinger B et al (2008) Bone mineral density changes during the menopause transition in a multiethnic cohort of women. J Clin Endocrinol Metab 93:861-868

27. Verhoeven AC, Boers M, te Koppele JM, van der Laan WH, Markusse HM, Geusens P, van der Linden S (2001) Bone turnover, joint damage and bone mineral density in early rheumatoid arthritis treated with combination therapy including high-dose prednisolone. Rheumatology 40:1231-1237

28. Guler-Yuksel M, Bijsterbosch J, Goekoop-Ruiterman YP, de VriesBouwstra JK, Hulsmans HM, de Beus WM et al (2008) Changes in bone mineral density in patients with recent onset, active rheumatoid arthritis. Ann Rheum Dis 67:823-828

29. Kanis JA, Burlet N, Cooper C, Delmas PD, Reginster JY, Borgstrom $\mathrm{F}$ et al (2008) European guidance for the diagnosis and management of osteoporosis in postmenopausal women. Osteoporos Int 19:399-428

30. Deodhar AA, Woolf AD (1996) Bone mass measurement and bone metabolism in rheumatoid arthritis: a review. Br J Rheumatol 35: 309-322

Publisher's note Springer Nature remains neutral with regard to jurisdictional claims in published maps and institutional affiliations. 